\title{
Successful Treatment of
}

Hepatocellular Carcinoma with

Transcatheter Arterial

Chemoembolization followed by
Radical Liver Transplantation in a
Patient with Severe Liver Damage

\author{
Hajime Nakamura ${ }^{a}$ Yutaka Kawano $^{\mathrm{b}}$ Koji Miyanishi ${ }^{\mathrm{a}}$ \\ Kazuma Ishikawa ${ }^{a}$ Tomohiro Kubo ${ }^{a}$ Shingo Tanaka ${ }^{a}$ Kohichi Takada \\ Masayoshi Kobune $^{c}$ Kohei Harada ${ }^{d}$ Norio Kawamura $^{e}$ \\ Tsuyoshi Shimamura $^{f}$ Hiromi Kanno-Okadag Junji Kato ${ }^{a}$ \\ aDepartment of Medical Oncology, Sapporo Medical University School of Medicine, \\ Sapporo, Japan; b Department of Gastroenterology, Health Sciences University of \\ Hokkaido Hospital, Sapporo, Japan; 'Department of Hematology, Sapporo Medical \\ University School of Medicine, Sapporo, Japan; dDivision of Radiology and Nuclear \\ Medicine, Sapporo Medical University Hospital, Sapporo, Japan; eDepartment of \\ Transplant Surgery, Graduate School of Medicine, Hokkaido University, Sapporo, Japan; \\ fDivision of Organ Transplantation, Central Clinical Facilities, Hokkaido University \\ Hospital, Sapporo, Japan; ${ }^{9}$ Department of Surgical Pathology, Hokkaido University \\ Hospital, Sapporo, Japan
}

\section{Keywords}

Hepatocellular carcinoma - Liver transplantation - Milan criteria - Transcatheter arterial chemoembolization · Downstaging 


\section{Case Reports in Oncology}

Case Rep Oncol 2019;12:289-296

DOI: $10.1159 / 000499703$

(c)

(C) 2019 The Author(s). Published by S. Karger AG, Base www.karger.com/cro

Nakamura et al.: Liver Transplantation after Successful Downstaging in a Patient with Severe Liver Damage

\begin{abstract}
Introduction: Liver transplantation for hepatocellular carcinoma (HCC) has been established as a curative therapy of underlying liver disease and cancer. However, the role of liver transplantation remains controversial for patients with HCC beyond Milan criteria. Case Presentation: A man in his 50s who was diagnosed as having two foci of HCC and advanced liver cirrhosis was referred to our hospital for further examination and treatment. Both foci of HCC were located in segment 8 of the liver and measured 39 and $9 \mathrm{~mm}$. Endoscopy showed esophageal varices that had a high risk of bleeding. After endoscopic ligation of the esophageal varices, he underwent transcatheter arterial chemoembolization (TACE) for downstaging of the advanced HCCs. No further liver deterioration was observed after TACE, and HCC staging was successfully downstaged to within the Milan criteria. One hundred ten days after TACE, he underwent liver transplantation; at 2.5 years after transplantation, he remains alive without HCC recurrence. Discussion/Conclusion: There are only a few treatment options available for patients with advanced HCC and severe liver damage. Multidisciplinary treatment such as locoregional treatments and prophylaxis of variceal bleeding may result in tumor downstaging, enabling radical liver transplantation without further exacerbation of liver damage.
\end{abstract}

(C) 2019 The Author(s)

Published by S. Karger AG, Basel

\section{Introduction}

Liver transplantation for hepatocellular carcinoma (HCC) has been established as a curative therapy of underlying liver disease and cancer. However, indications for liver transplantation in Japan include only HCC patients with severe liver damage, such as Child-TurcottePugh class C, 3 tumors not more than $3 \mathrm{~cm}$ in diameter or a single tumor not more than $5 \mathrm{~cm}$ in diameter. These Milan criteria for HCC treatment are well known as indications of liver transplantation in Japan [1]. We describe a case of advanced HCC complicated with severe liver damage and esophageal varices that was treated with transcatheter arterial chemoembolization (TACE) without exacerbation of liver damage, followed by radical liver transplantation.

\section{Case Report}

A man in his 50 s was transferred by ambulance to a nearby physician because of rupture of gastric varices. At that time, he was diagnosed as having cirrhosis after chronic type B hepatitis and had been followed up with by the physician afterward. He was suspected to have developed HCC based on the imaging examination in December 2014, and he was referred to our hospital in March 2015 for further examination and treatment.

At the time of the first visit, his consciousness was clear, but he had a flapping tremor. He presented with notable conjunctival yellowing. His abdomen was flat and soft without tenderness. Laboratory test results indicated pancytopenia, a prolonged prothrombin time-international normalized ratio, hypoalbuminemia, and jaundice. The result of the indocyanine green retention test was as high as $36.5 \%$. The alpha-fetoprotein level was within the normal range 


\section{Case Reports in Oncology}

Case Rep Oncol 2019;12:289-296

DOI: $10.1159 / 000499703$

2019 The Author(s). Published by S. Karger AG, Basel www.karger.com/cro

Nakamura et al.: Liver Transplantation after Successful Downstaging in a Patient with Severe Liver Damage

of $2.8 \mathrm{ng} / \mathrm{mL}$, but the protein induced by vitamin K absence/antagonist-II (PIVKA-II) level was as high as $163 \mathrm{mAU} / \mathrm{mL}$. He had cirrhosis after chronic type B hepatitis. The nucleotide analog (entecavir) was already prescribed by the previous physician, and the serological test result was positive for hepatitis B virus DNA (Table 1). Abdominal dynamic computed tomography (CT) showed that the liver edge was blunt, and the surface was irregular. A 39-mm nodule, which was enhanced in the arterial phase and slightly washed out in the equilibrium phase, was located in segment 8 of the liver. Moreover, prominent splenomegaly, collateral circulation, and a small amount of ascites in the pelvis were confirmed (Fig. 1a, b). In gadoliniumethoxybenzyl-diethilene-triamine-pentaacetic acid-enhanced dynamic magnetic resonance imaging, the 39-mm nodule, which was enhanced in the early phase and had a low signal in the hepatocyte phase, was also observed in segment 8 of the liver. Aside from this tumor, a small nodule measuring $9 \mathrm{~mm}$ in diameter showed the same contrast pattern in segment 8 of the liver (Fig. 1c, d). CT during arterial portography showed perfusion defects in the 2 HCCs, and CT during arteriography showed hypervascular tumors in the early phase and peripheral enhancement in the late phase, which was consistent with typical HCC contrast patterns (Fig. 1e-g). These HCCs were enhanced simultaneously during arteriography of A8, and obvious vascular invasion was not observed (Fig. 1h, i). No other lesions suspected of HCC were found in the liver. Upper gastrointestinal endoscopy revealed high-risk esophageal varices (Lm, F2, Cb, RC-sign [+]).

On the basis of these findings, we diagnosed the patient as having HCCs (cT3N0M0, clinical stage III) with severe liver damage. Regarding liver damage, the Child-Turcotte-Pugh classification was class C (11 points), and the Model for End-Stage Liver Disease score was 16 points, suggesting that he was a candidate for liver transplantation. Although there were 2 HCCs, one of them had a diameter exceeding $3 \mathrm{~cm}$, which deviated from the Milan criteria. When applying the HCC therapy algorithm in Japan to this patient's comprehensive disease condition, only palliative care was recommended. Therefore, to determine treatment other than palliative care, we consulted with a transplant surgeon. As both HCCs were perfused with A8, locoregional therapy could be conducted to downstage HCC and maintain the liver reserve. Moreover, since this patient was relatively young, and his performance status was good, we considered liver transplantation as a curative treatment for HCC and sever liver damage. After confirming the wishes of the patient and his family, we decided to perform TACE following radical liver transplantation. Before these treatments, endoscopic varicocele ligation was performed for the esophageal varices. After confirming the disappearance of varices, lipiodol TACE was performed in segment 8 of the liver with $20 \mathrm{mg}$ of epirubicin and gelatin sponges. There were neither postoperative complications nor changes in the Child-Turcotte-Pugh score. The PIVKA-II level decreased from 163 to $33 \mathrm{mAU} / \mathrm{mL}$ after TACE.

Abdominal dynamic CT on day 60 after TACE showed sufficient lipiodol deposition in the 2 HCCs in segment 8, but a tiny lipiodol deposit-free area remained (Fig. 1j, k). On the basis of these findings, we determined that the progress of HCCs was successfully downstaged within the Milan criteria, and this patient underwent liver transplantation 110 days after TACE. Surgery was performed using a left lobe graft (graft weight, $298 \mathrm{~g}$; graft-to-standard liver volume ratio, 25.8\%). The operative time was $13 \mathrm{~h}$ and $53 \mathrm{~min}$, and the bleeding volume was $7,885 \mathrm{~mL}$.

Pathologically, most of the tumor was successfully treated by TACE, except for the small remaining HCC where lipiodol deposition was insufficient. The smaller HCC which was $9 \mathrm{~mm}$ 


\section{Case Reports in Oncology}

Case Rep Oncol 2019;12:289-296

DOI: $10.1159 / 000499703$

(C) 2019 The Author(s). Published by S. Karger AG, Base www.karger.com/cro

Nakamura et al.: Liver Transplantation after Successful Downstaging in a Patient with Severe Liver Damage

before TACE was completed (Fig. 2a, b). Postoperatively, hyperbilirubinemia and ascetic accumulation due to small-for-size syndrome were temporarily observed but gradually improved after enlargement of the graft volume. This patient was discharged on day 35 after liver transplantation. Two and half years after transplantation, he remains alive without HCC recurrence or liver deterioration.

\section{Discussion}

Liver transplantation for HCC was formerly conducted on unresectable advanced HCC, but the outcome was poor because of high recurrence within a few years postoperatively [2]. Based on these dismal outcomes, the Milan criteria (a single HCC not more than $5 \mathrm{~cm}$ or up to 3 HCCs not more than $3 \mathrm{~cm}$ ) have been adopted worldwide for the selection of patients with HCC for liver transplantation, and a good outcome is achieved in patients who fulfill these criteria [3].

Some patients with advanced HCC beyond the Milan criteria were reported to achieve long-term survival after liver transplantation, and these reports have led to the development of expanded criteria worldwide [4]. University of California San Francisco criteria (a single tumor less than $6.5 \mathrm{~cm}$, maximum of 3 total tumors less than $4.5 \mathrm{~cm}$, and cumulative tumor size less than $8 \mathrm{~cm}$ ) and Asian criteria (tumor diameter not more than $5 \mathrm{~cm}$, number of lesions less than 7 , and no gross vascular invasion) are well known as extended criteria $[5,6]$. Therefore, the Milan group (Mazzaferro et al.) also created a new up-to-seven criteria ( 7 is the sum of the size of the largest tumor [in $\mathrm{cm}$ ] and the number of tumors), and the authors reported that patients who met these criteria achieved a 5-year overall survival of 71.2\% (64.2-77.0) [7]. Despite good outcomes overseas, liver transplantation was limited to the Milan criteria in Japanese patients with advanced HCC.

In recent years, locoregional therapy, such as TACE or radiofrequency ablation, for HCC downstaging within the Milan criteria has been reported. Yao et al. reported encouraging results of patients with HCC beyond the Milan criteria for liver transplantation [8]. However, aside from our case, most patients in this study had a Child-Turcotte-Pugh score less than 10 (Child-Turcotte-Pugh classes A and B). As even locoregional treatment causes liver damage to some extent, effective and safe treatment of HCC in patients with severe liver damage, especially Child-Turcotte-Pugh class $\mathrm{C}$, remains to be seen.

In our case, there were some advantages for downstaging treatment of HCC. One advantage was the location of the two HCCs. As these two HCCs were located within the same perfused area of A8, TACE could minimize the adverse effect of liver damage, resulting in a sustained Child-Turcotte-Pugh score. Additionally, prophylactic treatment of esophageal varices before locoregional TACE would also decrease the risk of mortality [9]. Finally, cooperation with transplant surgery could lead to a prompt intervention, resulting in successful liver transplantation.

In conclusion, TACE following liver transplantation with sustained liver damage is a feasible treatment option for severe liver damage patients with HCC beyond the Milan criteria in terms of its safety and efficacy. Regarding management of liver disease and treatment of advanced HCC, multidisciplinary treatment could have a beneficial result in tumor downstaging, enabling radical liver transplantation without further exacerbation of liver damage. 


\section{Statement of Ethics}

Written informed consent for publication was obtained from the patient.

\section{Disclosure Statement}

The authors have no conflicts of interest to declare.

\section{References}

1 Kokudo N, Hasegawa K, Akahane M, Igaki H, Izumi N, Ichida T, et al. Evidence-based Clinical Practice Guidelines for Hepatocellular Carcinoma: The Japan Society of Hepatology 2013 update (3rd JSH-HCC Guidelines). Hepatol Res. 2015 Jan;45(2):45.

2 Iwatsuki S, Gordon RD, Shaw BW Jr, Starzl TE. Role of liver transplantation in cancer therapy. Ann Surg. 1985 Oct;202(4):401-7.

3 Mazzaferro V, Regalia E, Doci R, Andreola S, Pulvirenti A, Bozzetti F, et al. Liver transplantation for the treatment of small hepatocellular carcinomas in patients with cirrhosis. N Engl J Med. 1996 Mar;334(11):693-9.

4 Endo I, Takeda K, Matsuo K, Kumamoto T, Tanaka K, Shimada H. [Current status of liver transplantation for hepatocellular carcinoma]. Gan To Kagaku Ryoho. 2010 Mar;37(3):417-23.

5 Yao FY, Ferrell L, Bass NM, Watson JJ, Bacchetti P, Venook A, et al. Liver transplantation for hepatocellular carcinoma: expansion of the tumor size limits does not adversely impact survival. Hepatology. 2001 Jun;33(6):1394-403.

6 Lee SG, Hwang S, Moon DB, Ahn CS, Kim KH, Sung KB, et al. Expanded indication criteria of living donor liver transplantation for hepatocellular carcinoma at one large-volume center. Liver Transpl. 2008 Jul;14(7):93545.

7 Mazzaferro V, Llovet JM, Miceli R, Bhoori S, Schiavo M, Mariani L, et al.; Metroticket Investigator Study Group. Predicting survival after liver transplantation in patients with hepatocellular carcinoma beyond the Milan criteria: a retrospective, exploratory analysis. Lancet Oncol. 2009 Jan;10(1):35-43.

8 Yao FY, Kerlan RK Jr, Hirose R, Davern TJ 3rd, Bass NM, Feng S, et al. Excellent outcome following downstaging of hepatocellular carcinoma prior to liver transplantation: an intention-to-treat analysis. Hepatology. 2008 Sep;48(3):819-27.

9 Lin CW, Chen YS, Lai CH, Perng DS, Weng HC, Hu JT, et al. Esophagogastric varices predict mortality in hospitalized patients with alcoholic liver disease in Taiwan. Hepatogastroenterology. 2010 MarApr;57(98):305-8. 


\section{Case Reports in Oncology}
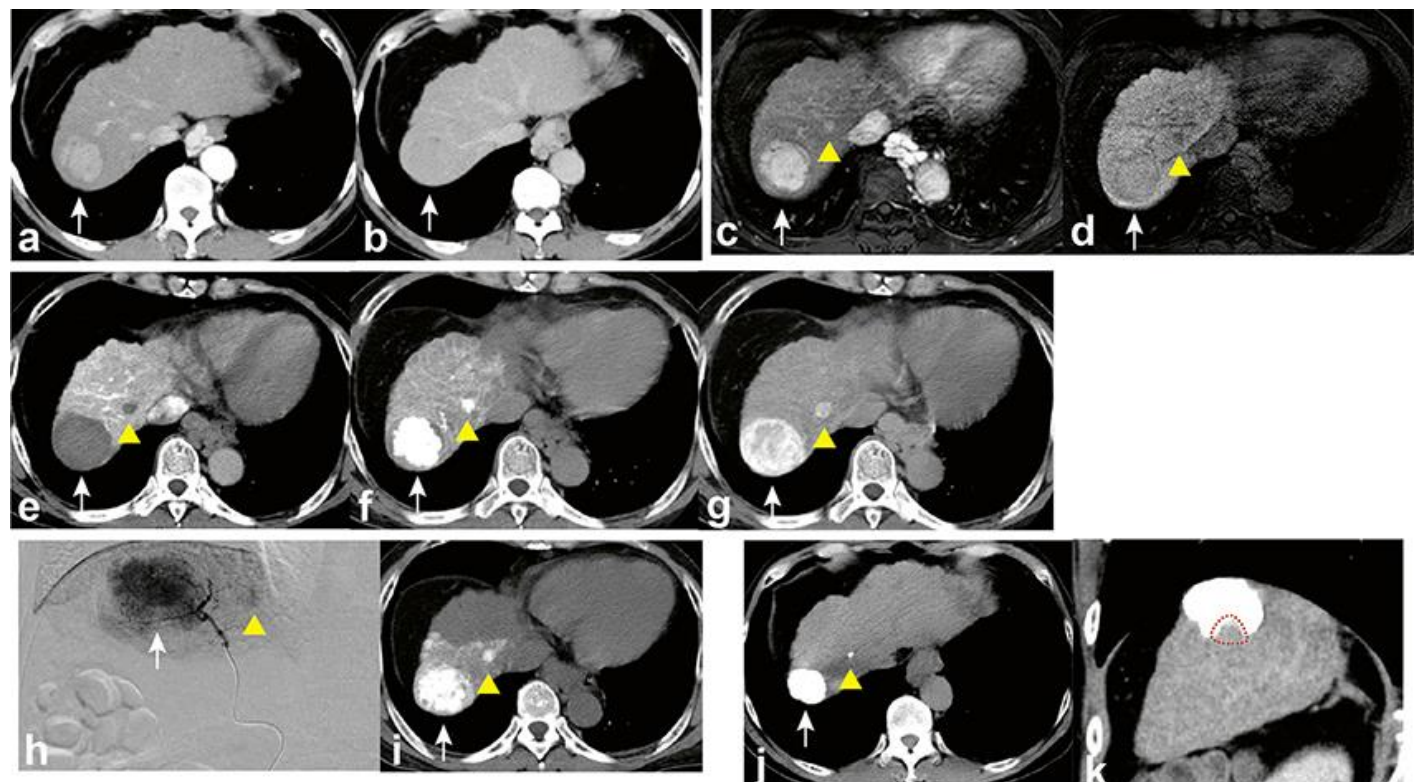

Nakamura et al.: Liver Transplantation after Successful Downstaging in a Patient with Severe Liver Damage

(c) 2019 The Author(s). Published by S. Karger AG, Basel www.karger.com/cro

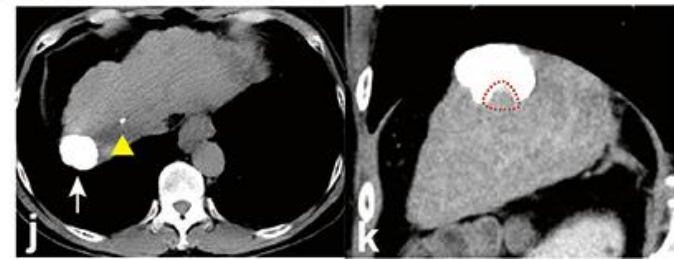

Fig. 1. Imaging results. (a) Arterial phase computed tomography (CT) showing a tumor (white arrow) in segment 8 with early enhancement. (b) Equilibrium phase CT showing that the tumor is slightly hypodense. (c) Arterial phase ethoxybenzyl magnetic resonance imaging (EOB-MRI) showing early enhancement of hepatocellular carcinomas (HCCs) measuring $39 \mathrm{~mm}$ (white arrow) and $9 \mathrm{~mm}$ (arrowhead) in diameter in segment 8 of the liver. (d) Hepatocellular phase EOB-MRI showing hypointensity of the 2 tumors. (e) CT during arterial portography showing a perfusion defect in the 2 HCCs. (f, g) CT during hepatic arteriography (CTHA) showed hypervascular tumors in the early phase (f) and peripheral enhancement in the late phase $(\mathbf{g})$. (h, i) Angiography showing both HCCs with enhancement during arteriography of A8 (h) and CTHA of A8 (i). (j, k) Enhanced CT findings 60 days after transcatheter arterial chemoembolization show sufficient lipiodol deposition in the 2 HCCs (j). However, a tiny lipiodol deposition-free area is also observed (red zone, $\mathbf{k}$ ). 


\section{Case Reports in Oncology}
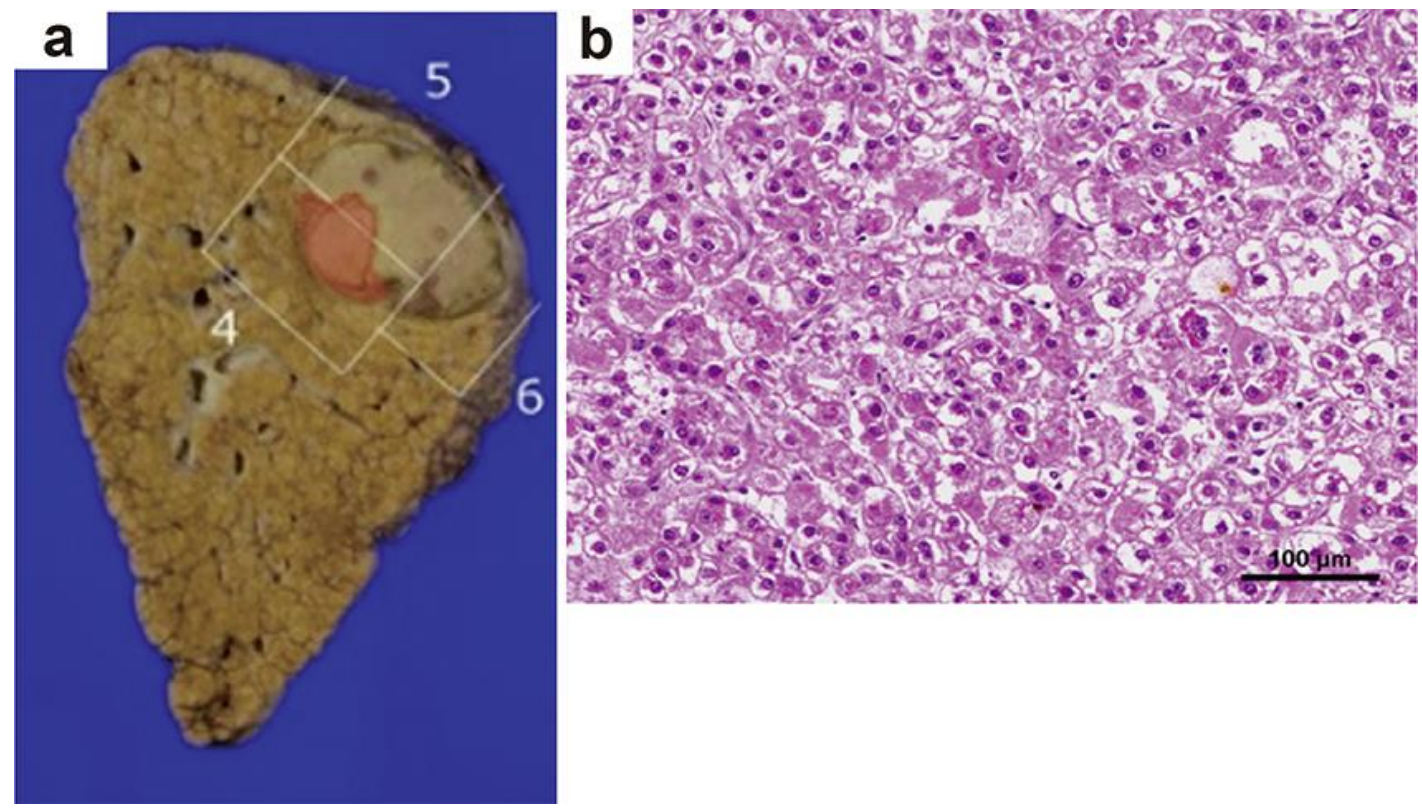

Fig. 2. Histopathological findings of the resected liver. (a) Photograph showing the removed section of the liver. Most of the tumor was successfully treated with transcatheter arterial chemoembolization, except for the small remaining viable hepatocellular carcinoma (HCC) (red zone). These lesions correspond with the computed tomography findings. (b) The viable tumor is a moderately differentiated HCC (hematoxylin and eosin staining). 
Nakamura et al.: Liver Transplantation after Successful Downstaging in a Patient with Severe Liver Damage

Table 1. Laboratory data on admission

\begin{tabular}{|c|c|c|c|c|c|}
\hline \multicolumn{2}{|c|}{ Peripheral blood } & \multicolumn{2}{|c|}{ Blood chemistry } & \multicolumn{2}{|c|}{ Tumor markers } \\
\hline WBC & $2,900 \mu \mathrm{L}$ & $\mathrm{TP}$ & $7.1 \mathrm{~g} / \mathrm{dL}$ & AFP & $2.8 \mathrm{ng} / \mathrm{mL}$ \\
\hline Neut & $82 \%$ & Alb & $3.4 \mathrm{~g} / \mathrm{dL}$ & PIVKA-II & $163 \mathrm{mAU} / \mathrm{mL}$ \\
\hline Lymph & $9 \%$ & T-Bil & $3.6 \mathrm{mg} / \mathrm{dL}$ & & \\
\hline Mono & $5 \%$ & D-Bil & $1.5 \mathrm{mg} / \mathrm{dL}$ & \multicolumn{2}{|c|}{ Serological test } \\
\hline Eos & $4 \%$ & AST & $42 \mathrm{IU} / \mathrm{L}$ & HBs Ag & + \\
\hline $\mathrm{RBC}$ & $453 \times 10^{4} / \mu \mathrm{L}$ & ALT & $28 \mathrm{IU} / \mathrm{L}$ & $\mathrm{HBs} \mathrm{Ab}$ & - \\
\hline $\mathrm{Hb}$ & $12.7 \mathrm{~g} / \mathrm{dL}$ & LDH & $301 \mathrm{IU} / \mathrm{L}$ & HBe Ag & - \\
\hline $\mathrm{Ht}$ & $37.5 \%$ & $\gamma$-GTP & $41 \mathrm{IU} / \mathrm{L}$ & $\mathrm{HBe} \mathrm{Ab}$ & + \\
\hline \multirow[t]{2}{*}{ Plt } & $3.6 \times 10^{4} / \mu \mathrm{L}$ & ALP & $349 \mathrm{IU} / \mathrm{L}$ & $\mathrm{HBc} \mathrm{Ab}$ & + \\
\hline & & CHE & 138 IU/L & HBV-DNA & nd \\
\hline \multicolumn{2}{|c|}{ Coagulation test } & BUN & $11 \mathrm{mg} / \mathrm{dL}$ & $\mathrm{HCV} \mathrm{Ab}$ & - \\
\hline PT & $50.7 \%$ & $\mathrm{Cr}$ & $0.64 \mathrm{mg} / \mathrm{dL}$ & RPR & - \\
\hline APTT & $37.2 \mathrm{~s}$ & $\mathrm{Na}$ & $142 \mathrm{mEq} / \mathrm{L}$ & TPHA & - \\
\hline FBG & $132 \mathrm{mg} / \mathrm{dL}$ & $\mathrm{Cl}$ & $108 \mathrm{mEq} / \mathrm{L}$ & & \\
\hline FDP & $<2.0 \mu \mathrm{g} / \mathrm{mL}$ & $\mathrm{K}$ & $3.4 \mathrm{mEq} / \mathrm{L}$ & & \\
\hline \multirow[t]{4}{*}{ AT-III } & $61 \%$ & $\mathrm{Ca}$ & $8.2 \mathrm{mg} / \mathrm{dL}$ & & \\
\hline & & IP & $1.9 \mathrm{mg} / \mathrm{dL}$ & & \\
\hline & & $\mathrm{NH}_{3}$ & $90.6 \mu \mathrm{g} / \mathrm{dL}$ & & \\
\hline & & ICG15min & $36.5 \%$ & & \\
\hline
\end{tabular}

WBC, white blood cell; Neut, neutrophil; Lymph, lymphocytes; Mono, monocytes; RBC, red blood cell; Hb, hemoglobin; Ht, hematocrit; Plt, platelet; PT, prothrombin time; APTT, activated partial thromboplastin time; FBG, fasting blood glucose; FDP, fibrinogen degradation product; AT-III, antithrombin III; TP, total protein; Alb, albumin; T-Bil, total bilirubin; D-Bil, direct bilirubin; AST, aspartate aminotransferase; ALT, alanine aminotransferase; LDH, lactate dehydrogenase; $\gamma$-GTP, gamma-glutamyl transferase; ALP, alkaline phosphatase; $\mathrm{CHE}$, cholinesterase; $\mathrm{BUN}$, blood urea nitrogen; $\mathrm{Cr}$, creatinine; $\mathrm{Na}$, sodium; $\mathrm{Cl}$, chloride; $\mathrm{K}$, potassium; Ca, calcium; IP, inducible protein; NH3, ammonia; ICG15min, indocyanine green retention test; AFP, alpha-fetoprotein; PVIKA-II, protein induced by vitamin K absence/antagonist-II; HBsAg, hepatitis B surface antigen; HBsAb, hepatitis B surface antibody; HBeAg, hepatitis B e-antigen; HBeAb, hepatitis B e antibody; HBcAb, hepatitis B core antibody; HBV-DNA, hepatitis B virus DNA; HCV Ab; hepatitis C antibody, $\mathrm{RPR}$, rapid plasma reagin; TPHA, treponema pallidum hemagglutination assay. 